\title{
Combatting mucosal melanoma: recent advances and future perspectives
}

\author{
Helen Tyrrell ${ }^{1}$ \& Miranda Payne*,1 \\ ${ }^{1}$ Oxford Cancer Centre, Oxford University Hospitals NHS Foundation Trust Oxford, Oxford, United Kingdom of Great Britain \& \\ Northern Ireland
}

*Author for correspondence: Miranda.Payne@ouh.nhs.uk

\section{Practice points}

- In any oral, sinonasal, vulval, vaginal, anal or rectal lesion, the possibility of mucosal melanoma should be considered and identified on histology.

- The only potentially curative option is complete surgical excision with negative margins. Extensive surgery with the potential to achieve negative margins should be weighed carefully against operative morbidity and the high risk of recurrent disease.

- Radiotherapy after surgery may improve local control, but there is no evidence it improves overall survival.

- Options for systemic therapy include immunotherapies and targeted treatments. Immunotherapy can be successful in mucosal melanoma, although response rates are lower than in cutaneous disease. Mucosal melanomas may harbor activating KIT mutations and other potentially targetable mutations, and therefore targeted treatments may be an option for selected patients.

- Clinical trials for patients with mucosal melanoma remain a key priority.

Mucosal melanomas are a rare subtype of melanoma and are associated with a particularly poor prognosis. Due to the rarity of the diagnosis, and the pace with which the management of cutaneous melanoma has evolved over recent years, there is little good evidence to guide management and evidence-based clinical guidelines are still in development in the UK. In this review we provide an overview of the management of mucosal melanoma, highlighting the critical differences between cutaneous and mucosal melanomas, before examining recent advances in the systemic treatment of this disease and likely future directions.

First draft submitted: 2 February 2018; Accepted for publication: 3 August 2018; Published online: 8 October 2018

Keywords: imatinib • ipilimumab • mucosal melanoma • nivolumab • pembrolizumab

In this review we provide an overview of the management of mucosal melanoma, highlighting the critical differences between cutaneous and mucosal melanomas (Table 1), before examining recent advances in the management of this disease and likely future directions. Due to the rarity of mucosal melanoma, and the pace with which the management of cutaneous melanoma has evolved over recent years, there is little good evidence to guide management and evidence-based clinical guidelines are still in development in the UK.

\section{Biology of melanocytes}

Melanomas are tumors arising from melanocytes, the cells whose primary function is to produce melanin pigment to protect the skin and eyes from sun damage. Most melanomas are therefore cutaneous. However, melanoma can also arise in the eye and from the mucosal surfaces. The most well-known function of melanocytes is to produce melanin and transport this, via membrane-bound organelles called melanosomes, to keratinocytes [2]. This occurs continuously during skin growth and differentiation, and the keratinocytes use them to form a protective shield around their DNA. As the mucosal tissues are not exposed to sunlight and so do not require UV protection, it is not entirely clear why melanocytes are found in the mucosal epithelium. It is not thought to be simply due to errors in migration from the neural crest during development, there is increasing evidence that melanocytes play a role in the immune system in these areas [2].

Melanin is produced from the amino acid tyrosine via a cascade of oxidation steps. This process is the main antimicrobial defense system in invertebrates. The cascade is triggered in response to lipopolysaccharides and

Future $\because \cdots$ Medicine 
Table 1. Comparison of mucosal and cutaneous melanoma.

\begin{tabular}{|c|c|c|}
\hline Comparates & Cutaneous melanoma & Mucosal melanoma \\
\hline Incidence & $\begin{array}{l}\text { Majority of melanomas, incidence increased } 20 \% \text { in } \\
20 \text { years [1] }\end{array}$ & $1.4 \%$ of all melanomas, stable over time [2] \\
\hline Median age at diagnosis & $65[1]$ & $70[3]$ \\
\hline Risk factors & $\begin{array}{l}\text { Sun exposure } \\
\text { Nevus count } \\
\text { Familial predisposition }\end{array}$ & None known \\
\hline 5-year survival for all stages at diagnosis & $\sim 90 \%[4,5]$ & $14 \%[4,5]$ \\
\hline Percentage with metastases at presentation & $5 \%[3]$ & $23 \%[5]$ \\
\hline Median OS from diagnosis of metastatic disease & 11.7 months [6] & 9.1 months [6] \\
\hline Response to immunotherapy & $\begin{array}{l}\text { ORR } 40.9 \% \text { with nivolumab alone } \\
\text { ORR } 60.4 \% \text { with combination immunothrapy [7] }\end{array}$ & $\begin{array}{l}\text { ORR } 23.3 \% \text { with nivolumab alone } \\
\text { ORR } 37.1 \% \text { with combination immunotherapy [7] }\end{array}$ \\
\hline Targetable mutations & BRAF V600 mutation in $\sim 50 \%[8-10]$ & $\begin{array}{l}\text { BRAF mutation in } 3-15 \% \\
\text { KIT alteration in } 7-17 \%[5,11-13]\end{array}$ \\
\hline
\end{tabular}

peptidoglycan, bacterial cell wall components and $\beta-1,3$-glucan, subunits of the cell walls of yeasts and molds. The melanin then traps the microorganism in capsules within the cell [14]. The intermediate compounds in the cascade, and free radicals produced by it, also directly kill microorganisms, probably due to disruption of their cell membranes. Melanin can also neutralize bacterial toxins [14]. In humans, many genetic defects leading to hypopigmentation are also associated with immune system defects [14]. Mediators of the human innate immune system can stimulate melanogenesis and melanocytes produce cytokines to drive the inflammatory response [15]. Melanocytes may also have an antigen-presenting function [2]. Data from studying US servicemen during the Vietnam war found that lighter skinned servicemen were more susceptible to both bacterial and fungal skin infections than African-American servicemen [14]. A role in the innate immune system potentially explains why melanocytes reside in the mucosal surfaces.

Melanocytes are also found in the brain where they produce neuromelanin. This is found throughout the brain, but at particularly high levels in dopaminergic neurones in the substantia nigra and locus coeruleus. They are thought to have a role in binding to reactive oxygen species and metals to prevent toxicity to these neurones [16]. Cases of primary CNS melanoma have been reported [17].

\section{Epidemiology \& etiology}

Mucosal melanoma is a rare disease, accounting for approximately $1.4 \%$ of all melanomas and only $0.03 \%$ of all new cancer diagnoses $[2,18]$. While there has been an almost $20 \%$ increase in the incidence of cutaneous melanoma since the 1990s [1], the incidence of mucosal melanoma has remained stable [18]. Mucosal melanomas arise from mucosal epithelium anywhere in the respiratory, genitourinary and GI tracts, but are most commonly found in three areas; the vulvovagina ( $18 \%$ of cases), the anorectum ( $24 \%$ of cases) and the oral cavity, nasal cavity or sinuses (55\% of cases) [3]. These are the mucosal surfaces arising from the ectoderm; mucosal melanomas of endodermal origin are extremely rare [4].

While the main risk factor for cutaneous melanoma is sun exposure, this is not a risk factor for mucosal melanomas which do not occur at sun-exposed sites. Indeed, no modifiable risk factor for mucosal melanomas has yet been identified. Studies suggest the average age of patients diagnosed with mucosal melanoma is slightly higher than for cutaneous melanoma, with a median age of diagnosis of 70 [3], compared with about 65 for cutaneous disease [1]. Oral mucosal melanoma is the exception, presenting in slightly younger patients [5]. The incidence is higher in females, due to the numbers of cases found in the female genital tract [2]. Mucosal melanoma is more common in Caucasians than other racial groups, although it represents a smaller proportion of all melanomas in Caucasians than other racial groups due to the greater incidence of cutaneous melanoma [2]. There is also no association between mucosal melanomas and nevus count or familial predisposition to melanomas [4].

Timely diagnosis can be complicated by the rarity of the tumor, the frequently occult site of origin, the fact that nearly half of mucosal melanomas are amelanotic compared with $<10 \%$ of cutaneous melanomas [19], and the multifocal growth pattern of up to one in five mucosal melanomas [19]. Patients with mucosal melanoma are more likely to present with regional or distant disease than patients with cutaneous melanoma. One study found that $55.5 \%$ of patients presented with localized disease, $21.5 \%$ with regional lymph node involvement and $23 \%$ 
with distant metastatic disease [5], whereas only $5 \%$ of patients with cutaneous melanoma have metastatic disease at presentation [3]. The most common sites of metastatic disease in mucosal melanoma are the liver and the lungs [5].

Mucosal melanoma is a disease with a very poor prognosis and most patients will develop incurable metastatic disease, irrespective of surgical excision. 5-year survival rates may be as low as $14 \%$, compared with $90 \%$ for cutaneous melanoma $[4,5]$ when all cases, regardless of stage are combined. Even when matched for stage at diagnosis, outcomes for mucosal melanoma remain inferior [4] and in patients with confirmed metastatic melanoma, a mucosal primary has been shown to be an independent poor prognostic factor by Kuk et al., who reported a single center retrospective study examining almost 3500 patients with melanoma diagnosed with metastatic disease from 2000 to 2013, including 237 patients with mucosal melanoma. Patients with cutaneous and uveal melanoma, and melanoma of unknown primary had similar survival, but patients with metastatic mucosal melanoma did least well, with a median survival of only 9.1 months [6]. The authors found no evidence that an anatomic site of origin affects survival [6], which is consistent with a series of over 700 patients diagnosed with mucosal melanoma published by Lian et al., who demonstrated that prognosis was similar regardless of anatomical site of origin [5]. They reported 1-year survival rates of 88,83 and $86 \%$ for nasopharyngeal and oral, gastrointestinal, and gynecological and urological mucosal melanoma, respectively, and 5-year survival rates of 27, 16 and 20\%, respectively [5].

\section{Staging}

No consensus on staging mucosal melanoma from different anatomical sites exists, and this complicates interpretation of the literature. Staging systems vary between, and sometimes within, anatomical sites of origin. For instance, the literature describing vulval melanoma uses both the AJCC (American Joint Committee on Cancer) cutaneous staging system and the International Federation of Gynecology and Obstetrics staging system, although the AJCC system has been demonstrated to be a better predictor of outcome [20]. Some anatomical sites, such as head and neck, have developed staging systems specifically for mucosal melanoma [21], which aim to reflect the very poor prognosis of this disease, while other tumor sites, such as anorectal, specifically exclude mucosal melanoma from their staging system [21].

A simplified staging system, which was originally developed for melanoma of the head and neck, can be applied to all cases of mucosal melanoma [22] but is not consistently used in clinical practice. The few clinical trials of systemic therapy from which patients with mucosal melanoma have not been excluded [23-26] usually, but not always [27], mandate use of the cutaneous staging system [28] in their eligibility criteria even for patients with mucosal disease, a system which is most flawed for the staging of nonmetastatic mucosal melanoma as it usually fails to reflect the often extensive lentiginous growth of these tumors and the disproportionately poor prognosis.

\section{Genetic testing}

A targetable driver mutation in codon V600 of the BRAF gene, which encodes the B-raf protein (a member of the Raf kinase family of growth signal protein transduction kinases), occurs in approximately $50 \%$ of cutaneous melanomas [29,30]. Identification of this mutation is clinically relevant, both for patients with confirmed metastatic melanoma and in patients at very high risk of recurrent disease (see Targeted therapy). While the BRAF gene can also be mutated in mucosal melanoma, the incidence of these mutations is much lower than in cutaneous melanoma. Data suggest it may occur in only $3-15 \%$ of all cases of mucosal melanoma $[5,11-13]$. In addition, a higher proportion of mutations observed in the $B R A F$ gene in mucosal melanoma affects regions of the $B R A F$ gene other than codon 600 , or are nonactivating mutations and therefore are not predicted to respond to targeted BRAF inhibition [12]. Despite the low incidence of targetable V600 mutations in mucosal melanoma, it is essential that $B R A F$ mutation testing is carried out in all cases of mucosal melanoma due to the existence of an effective and licensed treatment option for a very small subset of patients (see Targeted treatment).

Mutations in KIT, which encodes a transmembrane receptor tyrosine kinase, can be identified in $7-17 \%$ of all patients with mucosal melanoma [5,11-13] but may occur as frequently as one in three patients with vulvovaginal melanoma [13]. The specific mutation identified can predict tumor response to targeted inhibition and KIT is already an established therapeutic target in other cancers, for example, gastrointestinal stromal tumors (GIST). Identification of a potentially targetable activating mutation in KIT in a patient with melanoma may facilitate entry into relevant clinical trials or permit consideration of palliative treatment with a KIT inhibitor, such as imatinib, in the metastatic setting (see Targeted treatment). Therefore KIT mutation analysis should be considered for all patients diagnosed with mucosal melanoma. Unfortunately, errors in the KIT gene in mucosal melanoma are 
heterogeneous, dispersed across multiple exons, and included mutations and amplifications $[31,32]$. The potential clinical relevance of the different mutations is not yet fully understood.

The majority of melanomas contain potentially actionable genetic mutations [33] and this offers scope for broadening targeted treatment in the future. Molecular analysis for mutations in other genes known to be mutated in melanoma is recommended if a patient with mucosal melanoma is being considered for a clinical trial.

\section{Presentation}

Presentation with mucosal melanoma is typically with symptoms caused by locally advanced tumors, although almost one in four patients will present having already developed distant metastatic disease [5]. For instance, mucosal melanoma of the oral cavity can present with a bleeding mass, ulceration or mucosal discoloration although over half of cases are an incidental finding identified during routine dental examination. Sinonasal mucosal melanoma often presents with obstruction, epistaxis or loss of smell. Vulvovaginal mucosal melanomas present with pruritus, vaginal bleeding or discharge, dyspareunia or a mass, and anorectal mucosal melanoma generally presents with bleeding, pain, a mass, tenesmus or change in bowel habit.

\section{Localized disease}

The only curative treatment for mucosal melanoma is surgical resection. Complete surgical resection of mucosal melanoma with negative pathological margins is associated with a better prognosis [20,34] but there is no evidence that radical resection improves survival [35-38].

Complete pathological resection can be a particular challenge in this disease due to the lentiginous, sometimes multifocal pattern of growth [19] and surgically challenging anatomical sites. Unfortunately, most patients still die of their disease regardless of surgical intervention, even if pathologically negative margins have been achieved [34]. Local recurrence is commonly within a year of primary surgery and although re-excision can be considered, generally it is quickly followed by disseminated disease [39,40]. The morbidity of extensive surgery, offering the potential for negative margins, must therefore be weighed carefully against the very high risk of future incurable disease recurrence. As most patients ultimately develop distant metastatic disease, quality-of-life is critical in decision making.

\section{Radiotherapy}

It has never been proved that radiotherapy after primary resection improves overall survival [41-43] but it may improve the rate of local disease control [41-44]. In a published review of practice spanning 20 years, 54 patients underwent sphincter-sparing surgery for anorectal mucosal melanoma and subsequently received hypofractionated radiotherapy. Overall survival was poor at $30 \%$, but local disease recurrence occurred in only $17 \%$ of patients and radiotherapy was well tolerated, with almost all patients retaining good sphincter function [42]. Similarly, a retrospective, nonrandomized series of 160 patients with nonmetastatic head and neck mucosal melanoma treated between 1980 and 2008 examined the pattern of recurrence observed in patients treated by surgery alone, compared with patients who received radiotherapy after their surgery. The probability of survival was unaffected by treatment modality. However patients who underwent both surgery and postoperative radiotherapy were less likely to experience local recurrence as their first disease-related event after primary treatment [41]. In unresectable, bulky or symptomatic disease, radiotherapy with palliative intent can also be considered.

\section{Systemic treatment of unresectable or metastatic disease Immunotherapy}

The management of patients with metastatic cutaneous melanoma has seen enormous progress over the last few years with the introduction of immunotherapy into routine use and is comprehensively described in many published reviews [45-47]. Monoclonal antibodies against both programmed cell death 1 (PD-1) with nivolumab and pembrolizumab, and against cytotoxic T-lymphocyte antigen 4, with ipilimumab, are licensed for use, are in routine clinical use in the UK and have changed the course of this disease for many patients [23-26]. A pooled analysis of almost 2000 patients with melanoma of all subtypes receiving ipilimumab in both trials and an expanded access program showed a median overall survival of only 9.5 months, but clearly demonstrated the potential for durable disease control even in the context of metastatic disease, with $21 \%$ of patients alive at 3 years and, most significantly, a plateauing of the survival curve [48]. Response rates improved with the introduction of anti-PD-1 monotherapy. Nivolumab (3 mg/kg every 2 weeks until progression) is associated with a $40 \%$ response rate with a 5.1 -month 
progression-free survival in metastatic melanoma [23] and a significant improvement in adverse events compared with ipilimumab. Similar results are seen with single-agent pembrolizumab $(2 \mathrm{mg} / \mathrm{kg}$ every 3 weeks $)$, with a median overall survival of 13.4 months and a 2 -year survival of $36 \%$ in a trial of pembrolizumab in patients who had previously received ipilimumab [49]. In another trial a response rate of $45 \%$ was reported in treatment-naive patients and a $33 \%$ response rate when those previously treated with ipilimumab was included [50]. The combination of ipilimumab (3 mg/kg for four doses) with nivolumab $(1 \mathrm{mg} / \mathrm{kg}$ for four doses followed by maintenance monotherapy with $3 \mathrm{mg} / \mathrm{kg}$ every 2 weeks) offers scope to improve still further on response rates, resulting in a median progression-free survival of 11.5 months, compared with 2.9 months for ipilimumab alone or 6.9 months for nivolumab monotherapy [51]. However, the high rates of grades 3 and 4 toxicity seen with combination immunotherapy, and the unexpectedly modest improvement on 3 -year survival of $58 \%$ for patients treated with combination immunotherapy compared with $52 \%$ for patients treated with nivolumab monotherapy [52], have complicated decision making in the clinic.

Pleasingly, many of the trials of these agents have not excluded patients with mucosal melanoma, although they have not reported the results from these patients separately due to their rarity. 2017 saw the publication of a pooled analysis of the outcomes of patients with metastatic mucosal melanoma treated in trials either with single-agent nivolumab or the combination of ipilimumab and nivolumab, comparing outcomes to those of patients with cutaneous melanoma. Six trials were included in the analysis; two Phase I studies of nivolumab, a Phase II trial of ipilimumab monotherapy versus combination ipilimumab and nivolumab, and three Phase III trials of nivolumab, either versus chemotherapy, ipilimumab monotherapy or combination ipilimumab and nivolumab. 86 patients with histologically confirmed metastatic mucosal melanoma were identified who had been treated with nivolumab and an additional 35 had received the combination of nivolumab and ipilimumab [7]. The pooled analysis confirmed that patients with metastatic mucosal melanoma can benefit from treatment with the immunotherapy regimes which are in routine clinical use for patients with metastatic cutaneous melanoma. However, the objective response rates (ORR) are lower. For nivolumab monotherapy the ORR in the pooled analysis was $23.3 \%$ in the mucosal melanoma population (95\% CI: 14.8-33.6\%), compared with $40.9 \%$ for patients with cutaneous disease (95\% CI: $37.1-44.7 \%)$. Treatment with the combination of ipilimumab and nivolumab was associated with an ORR of only $37.1 \%$ (95\% CI: $21.5-55.5 \%$ ), compared with $60.4 \%$ observed in patients with metastatic cutaneous melanoma (95\% CI: 54.9-65.8\%) [7].

Another study identified 35 patients with mucosal melanoma treated with anti-PD-1 monotherapy (nivolumab or pembrolizumab) either within a clinical trial, expanded access program or through standard clinical practice [53]. Seven patients overlapped between these two publications [7,53]. Of 35 patients, $23 \%$ had an objective response and $57 \%$ had only progressive disease, with a median progression-free survival of 3.9 months [53]. Of those who had a partial or complete response, the median duration of response was 12.9 months. Both papers reported that treatment was well tolerated and very few patients discontinued due to toxicity $[7,53]$. Indeed D'Angelo $e t$ al. noted that the incidence of treatment-related adverse events appeared marginally lower in patients with mucosal melanoma than for patients with cutaneous melanoma [7] for reasons which are not known, but may be related to shorter duration of treatment.

It is not known why response rates to immunotherapy are lower in mucosal melanoma. Possibly it results from the lower proportion of patients with mucosal melanoma in the pooled analysis who had high levels of PD-L1 expression, defined as $>5 \%$, in their tumors. Only 25 out of $121(21 \%)$ of mucosal melanoma patients in these trials had high PD-L1 expression, compared with 348 out of 991 (35\%) of cutaneous melanoma patients [7], but in the pooled analysis, even patients with low tumor PD-L1 expression $(<5 \%)$ demonstrated an ORR of $33 \%$ to combination immunotherapy. To date there is no evidence to support a potential role for tumor PD-L1 expression as a biomarker for response either to monotherapy or combination immunotherapy in metastatic mucosal melanoma, a situation which mirrors that of cutaneous melanoma [52]. Alternatively it has been hypothesized that response to immunotherapy correlates with the number of mutations in a tumor and therefore the antigen load. As mucosal melanoma is not induced by sun damage, it would be predicted to be associated with a lower mutational burden than cutaneous melanoma and this could account for the poorer response to immunotherapy. Johnson et al. undertook comprehensive genomic profiling on over 2000 melanoma samples, of which $2 \%$ were mucosal in origin. Tumor mutational burden in mucosal melanomas was found to be markedly lower than in cutaneous melanomas [13]. Consistent with this, a study that performed either whole genome or whole exome sequencing on ten mucosal melanoma samples found that mucosal melanoma is associated with a five- to ten-fold lower rate of mutations compared with cutaneous melanomas [4]. The mutational burden found in the mucosal melanomas in 
this study was comparable to that seen in breast and prostate cancers, which are also not associated with known environmental carcinogens. They did, however, find that their samples had nearly four-times as many structural variants as seen in cutaneous melanoma, such as chromosomal aberrations and copy number alterations. The cause of the chromosomal instability in these samples was unclear [4].

Studies suggest that the presence of tumor-infiltrating lymphocytes (TILs) may be an important predictor of response to anti-PD-1 agents [54] but little is published about the prevalence of TILs in mucosal melanomas [53]. One Chinese study of pathological analysis of 82 cases of oral mucosal melanoma found that the presence of TILs was associated with a lower risk of distant metastases [55].

Numerous new immunotherapy agents are in development, either as monotherapy or in combination with established treatments, including LAG3 antagonists and OX40 agonists. Permitting patients with mucosal melanoma to be included in the clinical trials of these drugs is essential to ensure this patient group has the potential to benefit from emerging agents.

In summary, with trial data to suggest a response rate of up to $37 \%$ with ipilimumab combined with nivolumab in metastatic mucosal melanoma, combination immunotherapy offers an evidence-based treatment option for patients of good performance status with this disease. However, potential toxicity is high, improvement in overall survival may not reflect the higher response rates, and evidence on durability of response in the rare mucosal patient subgroup is lacking. Patients must be counselled on the risks of treatment, and clinicians and patients should be encouraged to consider quality-of-life factors in decision making. For less fit patients, or those whose preference is to avoid a high risk of potentially serious side effects, anti-PD-1 monotherapy also has evidence to support its efficacy in metastatic mucosal melanoma.

\section{Targeted therapy}

Nearly half of cutaneous melanomas harbor activating $B R A F$ mutations and therefore can be treated with BRAF kinase inhibitors such as dabrafenib (150 mg twice daily), often in combination with an MEK inhibitor, such as trametinib ( $2 \mathrm{mg}$ once daily). The targeted treatment of metastatic melanoma has been widely reviewed before [8-10] and will not be repeated in detail here. In a Phase III trial of 704 patients the combination of a BRAF and an MEK inhibitor was associated with an ORR of $64 \%$ and a median progression-free survival of 11.4 months, compared with a response rate of $51 \%$ and median progression-free survival of 7.3 months for a BRAF inhibitor alone [56]. Mucosal melanomas rarely have mutations in $B R A F$ and therefore this is not usually an option for these patients. However, all mucosal melanoma patients should be tested for $B R A F$ mutations and if an activating $B R A F$ mutation is found in a patient with mucosal melanoma, then the combination of a BRAF and an MEK inhibitor should be considered.

Mucosal melanomas do sometimes have activating mutations in KIT and therefore tyrosine kinase inhibitors, such as imatinib and dasatinib, targeting the aberrant protein gene product have been trialed with some positive results. While KIT testing is not currently routinely available in all centers across the UK, mutation analysis is advised in mucosal melanoma, as a small minority of patients will have targetable mutations and may potentially benefit from treatment, ideally within the context of a clinical trial. A Phase II trial of imatinib in 24 patients with either $K I T$-mutated or $K I T$-amplified tumors in mucosal, acral or chronically sun-damaged melanoma found that it was effective in patients with KIT-mutated tumors, but not in those where the gene was amplified only. Of 13 patients with $K I T$ mutations, seven $(54 \%)$ had a partial response. Of the 24 patients treated, 17 had mucosal melanoma and of these 11 had a KIT mutation. The seven patients in the study who had a partial response all came from the subgroup with mucosal melanomas with KIT mutations, meaning within this group the response rate was $7 / 11(64 \%)$ [31].

Another trial of imatinib in the same melanoma subtypes in patients with KIT mutations or amplifications treated 25 patients [57]. Two patients experienced complete responses and four had partial responses. In four of these six cases the response was durable, lasting more than a year. Of the 25 evaluable cases, 13 had mucosal melanoma one of these had a complete response and two a partial response, giving a response rate of $3 / 13(23.1 \%)$. Another study looked at 43 patients with melanoma with KIT aberrations and found a partial response rate of $23.3 \%$ and a disease control rate of $53.5 \%$ [58].

A trial of dasatinib, intended specifically to target mutations in exon 11 , as first-line treatment in mucosal melanomas with KIT mutations gave disappointing results, with response rates of $18.2 \%$ and no difference in outcomes between patients with exon 11 KIT mutation than with alternative mutations [59]. The authors concluded that imatinib should remain the first-choice treatment option for patients with a KIT mutation. In a Phase II trial, 
patients with all types of melanomas with KIT mutations or amplifications, who had progressed on previous KIT inhibitor therapy, were given second-line nilotinib. Two out of 11 patients achieved a partial response, including a lady with vulvar melanoma who had an ongoing response at 37.5 months [32], and hence, the possibility of treating with further tyrosine kinase inhibitors after progression on one drug should not be discounted.

Unfortunately, targeted treatment for $K I T$-mutated mucosal melanoma does not offer the clinical reliability observed with $B R A F$-targeted therapy in cutaneous melanoma. Errors in the KIT gene are heterogeneous, dispersed across multiple exons, and include mutations and amplifications [31,32], but clinical benefit from targeting this mutated protein appears restricted to specific mutations. Within the context of clinical trials, activating mutations or amplifications in exons 11 and 13 of the KIT gene have most frequently been associated with durable clinical benefit from KIT inhibitors [57,58] while other patients have derived no clinical benefit. In four patients reported by Hodi et al. [31] there was a co-existing NRAS mutation at the start of treatment and all of these patients progressed on imatinib. It is possible that one mechanism of primary resistance to imatinib may be mediated by activation of this pathway raising the future possibility that a combination of an MEK and KIT inhibitor could be effective in these patients [31]. Carvajal et al. [57] noted that all six responses to KIT inhibition occurred in tumors with L576P or K642E mutations. There were also patients in this study with the V654A and D820Y mutations, known to confer resistance to imatinib in GIST, and these patients both progressed on imatinib. Overall response rates are lower than that seen in GIST with KIT mutations and further clinical trials are required for us to be able to usefully exploit the KIT gene as a therapeutic target in mucosal melanoma [57].

NRAS mutations have been found in $15-20 \%$ of melanomas, and this is fairly consistent across all melanoma subtypes, including mucosal [60]. NRAS mutations are associated with a poor prognosis and are a potential cause of BRAF inhibitor resistance when BRAF is mutated as well. Trials are ongoing of drugs targeting the MAPK pathway and the phosphatidylinositide 3-kinase (PI3K) pathway, which work downstream of NRAS. MEK inhibitors, which affect the MAPK pathway, may be effective in patients with NRAS mutations and trials combining these with other agents are also ongoing. The MEK inhibitor binimetinib has been shown to give a response rate of $20 \%$ in a Phase II trial of 30 patients with NRAS-mutated melanoma [61] and a Phase III trial is ongoing. No trial has yet demonstrated an improvement in overall survival following treatment with MEK inhibitor monotherapy. Trials are also ongoing combining MEK inhibitors with PI3K/AKT inhibitors or CDK4/6 inhibitors [60]. Given that these genes can be mutated in mucosal melanoma, it is to be hoped that future clinical trials do not exclude this rare subgroup of patients.

\section{Chemotherapy}

Until the arrival of checkpoint inhibitors and targeted treatments in the last decade, dacarbazine chemotherapy had been the standard-of-care for metastatic melanoma, as no combination chemotherapy regimens had been shown to give a further improved survival advantage [62]. Conventional chemotherapy is now rarely given in melanoma, but there is renewed interest in the possibility of combining chemotherapy with immunotherapy, and several trials are underway [63]. There is evidence that immunotherapy can be safely administered in combination with chemotherapy as some patients in early studies with ipilimumab received it in combination with dacarbazine [64,65]. It is hypothesized that cytotoxic chemotherapy may potentiate the immune response by causing immunogenic cell death. Should this approach show promise, it would be of great interest to include the mucosal melanoma population within future studies.

\section{Adjuvant systemic therapy after primary resection}

Although adjuvant therapy after resection of high-risk cutaneous melanoma is not yet standard-of-care in the UK, recently published clinical trials are likely to prompt this to change.

Until 2017, the only published randomized evidence supporting adjuvant therapy after resection of mucosal melanoma was from Lian et al. They randomized 189 patients with resected mucosal melanoma to either observation, or 1 year of high-dose IFN- $\alpha 2 \mathrm{~b}\left(15 \times 10^{6} \mathrm{U} / \mathrm{m}^{2} / \mathrm{d}\right.$ IFN- $\alpha 2 \mathrm{~b}$ D $1-5$ for 4 weeks followed by $9 \times 10^{6} \mathrm{U}$ IFN- $\alpha 2 b$ three-times a week for 48 weeks), or three weekly chemotherapy with temozolomide $\left(200 \mathrm{mg} / \mathrm{m}^{2} / \mathrm{d}\right.$ D1-5) plus cisplatin $\left(75 \mathrm{mg} / \mathrm{m}^{2}\right.$ divided over 3 days) for six cycles [27]. Prognosis was poor across all groups. However, adjuvant therapy was associated with a trend toward improved survival (relapse-free survival 5.4, 9.4 and 20.8 months, respectively), which reached statistical significance ( $<<0.001$ for IFN- $\alpha 2 b$ and for chemotherapy). The median overall survival in the observation arm was 21.2 months (95\% CI: 15.8-26.6 months), 40.4 months for patients who had received IFN- $\alpha 2 \mathrm{~b}$ (95\% CI: 32.5-48.3 months) and 48.7 months for patients who had 
received chemotherapy (95\% CI: 41.8-55.6 months). Numbers of patients included in this trial are impressive, given the rarity of the diagnosis, but extrapolation of the results are difficult within the current clinical environment. A trial favoring adjuvant chemotherapy over immunotherapy in the treatment of melanoma is discordant with oncological principals of systemic adjuvant therapy. The chemotherapy regime of temozolomide plus cisplatin has no proven survival benefit in the palliative treatment of metastatic mucosal melanoma and both regimes predate the introduction of currently established systemic treatments for metastatic cutaneous melanoma.

These results have been superseded by the more recent publications of immunotherapy trials in the adjuvant treatment of melanoma. The cytotoxic T-lymphocyte antigen 4-specific monoclonal antibody ipilimumab is licensed as adjuvant therapy in stage III melanoma, based on the results from a Phase III study published in 2015 in which over 900 patients with cutaneous melanoma were randomized to either ipilimumab $10 \mathrm{mg} / \mathrm{kg}$ for four doses, followed by the same dose every 3 months for 3 years, or placebo. Treatment with ipilimumab was associated with a $10 \%$ increase in 3-year recurrence-free survival, and median recurrence-free survival was 26.1 months in the ipilimumab-treated group versus 17.1 months in the control arm [66]. Concerns over the high incidence of serious side effects ( $52 \%$ of patients interrupted treatment due to toxicity), and anticipation of imminent publication of results from adjuvant anti-PD-1 trials, precluded this regime from adoption into routine practise in the UK. Patients with mucosal melanoma were specifically excluded, but fortunately were permitted to enter the more recently published randomized, double-blind trial comparing 1 year of adjuvant ipilimumab $10 \mathrm{mg} / \mathrm{kg}$ for four doses, followed by the same dose every 3 months, with the PD-1 inhibitor nivolumab $3 \mathrm{mg} / \mathrm{kg}$ every 2 weeks in over 900 patients with resected stage III or IV melanoma. Nivolumab was associated with a significant improvement in 1 -year recurrence-free survival of $70.5 \%$, compared with $60.8 \%$ with ipilimumab. Nivolumab was also significantly better tolerated with only a $14.4 \%$ incidence of grade 3 or 4 adverse events compared with $45.9 \%$ in the ipilimumab group [67]. A total of 29 patients with mucosal melanoma were included in this study, staged according to the 2009 AJCC melanoma staging system [28] of whom 16 were randomized to nivolumab.

Such small numbers of patients with mucosal melanoma preclude meaningful subgroup analysis. Although further trials in patients with high-risk melanoma are underway examining potential adjuvant combination immunotherapy regimes and optimal scheduling, due to the rarity of mucosal melanoma, and the scale of the trials required to demonstrate a clinically meaningful adjuvant benefit, this is not a situation that is likely to be improved on. Adjuvant decisions for patients with resected mucosal melanoma will need to consider the existing, predominantly cutaneous, adjuvant evidence base, the lower response rates to immunotherapy observed in metastatic mucosal melanoma and the disproportionately high risk of incurable recurrence that these patients face.

In the future, targeted therapy may offer an alternative adjuvant therapy option for a small minority of patients with resected mucosal melanoma. Patients who have undergone resection of a GIST tumor harboring a mutation in the KIT gene may be offered imatinib in the adjuvant setting and this does significantly reduce recurrence rates [68]. Given similar mutations can be identified in up to a third of patients with mucosal melanoma [5,11-13], adjuvant use of KIT inhibitors may offer potential for modifying the course of the disease. Whether patient numbers will ever be sufficient for this to be established within the context of a randomized clinical trial is very doubtful.

Occasionally, mucosal melanoma will be identified to harbor an activating mutation in codon 600 of the $B R A F$ gene [5,11-13]. 2017 saw the publication of a randomized, double-blind, placebo-controlled trial of adjuvant dabrafenib $150 \mathrm{mg}$ twice daily with trametinib $2 \mathrm{mg}$ once daily in 870 patients with resected stage III cutaneous melanoma possessing a BRAF V600E or V600K mutation. Adjuvant treatment with dabrafenib and trametinib was associated with a statistically significant improvement in relapse-free survival at 3 years of $58 \%$, compared with $39 \%$ for placebo, and an overall survival at 3 years of $86 \%$ for those receiving the combination therapy, compared with $77 \%$ for controls [69]. Extrapolation of these results to the very small minority of patients with mucosal melanoma harboring the same activating mutations offers future potential for modifying the course of their disease, a possibility of critical clinical significance given their greater risk of death from metastatic disease.

\section{Conclusion \& future perspective}

Patients diagnosed with mucosal melanoma represent a rare subgroup of the melanoma patient population. Although the principles of care are the same, there are critical differences between mucosal and cutaneous melanoma, which must influence future clinical decision making. While complete surgical excision offers the only prospect of cure, the challenging anatomical sites present a high risk of surgical morbidity and most patients still develop incurable metastatic disease. Systemic treatment with immunotherapy or targeted therapy can offer scope for modifying the course of the disease but response rates are lower and clinical research remains a priority, particularly as there are 
critical genetic differences which have not yet been fully exploited. Evidence-based UK clinical guidelines for the management of patients with mucosal melanoma are in development and aim to minimize inequality in care. In the meantime, sufficient evidence exists to recommend use of some of the currently available systemic therapies. The rarity of the diagnosis will ensure that large-scale mucosal melanoma trials are unlikely ever to be feasible. Including patients with metastatic mucosal melanoma in trials of new agents for the treatment of melanoma will allow us gradually to expand our evidence-base and ensure that we identify those regimes which offer genuine potential for this small but important patient group.

\section{Financial \& competing interests disclosure}

M Payne discloses funding for attendance at conferences, speakers' fees, and honoraria for participation in advisory boards from GSK, BMS, Novartis, Incyte, and MSD. The authors have no other relevant affiliations or financial involvement with any organization or entity with a financial interest in or financial conflict with the subject matter or materials discussed in the manuscript. This includes employment, consultancies, honoraria, stock ownership or options, expert testimony, grants or patents received or pending, or royalties.

No writing assistance was utilized in the production of this manuscript.

\section{Open access}

This work is licensed under the Attribution-NonCommercial-NoDerivatives 4.0 Unported License. To view a copy of this license, visit http://creativecommons.org/licenses/by-nc-nd/4.0/

\section{References}

Papers of special note have been highlighted as: $\bullet \bullet$ of considerable interest

1. UK CRUK. Skin cancer incidence statistics. www.cancerresearchuk.org/health-professional/cancer-statistics

2. Mihajlovic M, Vlajkovic S, Jovanovic P, Stefanovic V. Primary mucosal melanomas: a comprehensive review. Int. J. Clin. Exp. Pathol. 5(8), 739-753 (2012).

3. Chang AE, Karnell LH, Menck HR. The National Cancer Data Base report on cutaneous and noncutaneous melanoma: a summary of 84,836 cases from the past decade. The American College of Surgeons Commission on Cancer and the American Cancer Society. Cancer 83(8), 1664-1678 (1998).

4. Furney SJ, Turajlic S, Stamp G et al. Genome sequencing of mucosal melanomas reveals that they are driven by distinct mechanisms from cutaneous melanoma. J. Pathol. 230(3), 261-269 (2013).

5. Lian B, Cui CL, Zhou L et al. The natural history and patterns of metastases from mucosal melanoma: an analysis of 706 prospectively-followed patients. Ann. Oncol. 28(4), 868-873 (2017).

6. Kuk D, Shoushtari AN, Barker CA et al. Prognosis of mucosal, uveal, acral, nonacral cutaneous, and unknown primary melanoma from the time of first metastasis. Oncologist 21(7), 848-854 (2016).

-• Clearly demonstrates the worse prognosis in mucosal melanoma compared with other melanoma subgroups.

7. D'Angelo SP, Larkin J, Sosman JA et al. Efficacy and safety of nivolumab alone or in combination with ipilimumab in patients with mucosal melanoma: a pooled analysis. J. Clin. Oncol.35(2), 226-235 (2017).

-. An excellent study, which shows the benefits of immunotherapy for patients with mucosal melanoma.

8. Griffin M, Scotto D, Josephs DH et al. BRAF inhibitors: resistance and the promise of combination treatments for melanoma. Oncotarget 8(44), 78174-78192 (2017).

9. Karoulia Z, Gavathiotis E, Poulikakos PI. New perspectives for targeting RAF kinase in human cancer. Nat. Rev. Cancer 17(11), 676-691 (2017).

10. Lim SY, Menzies AM, Rizos H. Mechanisms and strategies to overcome resistance to molecularly targeted therapy for melanoma. Cancer 123(S11), 2118-2129 (2017).

11. Öztürk Sari Ş, Yilmaz I, Taşkin O et al. BRAF, NRAS, KIT, TERT, GNAQ/GNA11 mutation profile analysis of head and neck mucosal melanomas: a study of 42 cases. Pathology 49(1), 55-61 (2017).

12. Lyu J, Wu Y, Li C et al. Mutation scanning of BRAF, NRAS, KIT, and GNAQ/GNA11 in oral mucosal melanoma: a study of 57 cases. J. Oral Pathol. Med. 45(4), 295-301 (2016).

13. Johnson DB, Carlson JA, Elvin JA et al. Landscape of genomic alterations (GA) and tumour mutational burden (TMB) in different metastatic melanoma (MM) subtypes. J. Clin. Oncol. 35, (No. 15 Suppl.) 9536-9536 (2017).

14. Mackintosh JA. The antimicrobial properties of melanocytes, melanosomes and melanin and the evolution of black skin. J. Theor. Biol. 211(2), 101-113 (2001).

15. Gasque P, Jaffar-Bandjee MC. The immunology and inflammatory responses of human melanocytes in infectious diseases. J. Infect. 71(4), 413-421 (2015). 
16. Plonka PM, Passeron T, Brenner M et al. What are melanocytes really doing all day long. . ? Exp. Dermatol. 18(9), $799-819$ (2009).

17. Mekni A, Braham E, Nouira K et al. Primary central nervous system malignant melanoma: report of 5 cases. Pathologica 99(3), 71-75 (2007).

18. Patrick RJ, Fenske NA, Messina JL. Primary mucosal melanoma. J. Am. Acad. Dermatol. 56(5), 828-834 (2007).

19. Carvajal RD, Spencer SA, Lydiatt W. Mucosal melanoma: a clinically and biologically unique disease entity. J. Natl Compr. Canc. Netw. 10(3), 345-356 (2012).

20. Seifried S, Haydu LE, Quinn MJ, Scolyer RA, Stretch JR, Thompson JF. Melanoma of the vulva and vagina: principles of staging and their relevance to management based on a clinicopathologic analysis of 85 cases. Ann. Surg. Oncol. 22(6), 1959-1966 (2015).

21. Edge S, Compton C. American Joint Committee on Cancer Cancer Staging Manual. Springer MA, USA (2009).

22. Ballantyne AJ. Malignant melanoma of the skin of the head and neck. An analysis of 405 cases. Am. J. Surg. 120(4), 425-431 (1970).

23. Robert C, Long GV, Brady B et al. Nivolumab in previously untreated melanoma without BRAF mutation. N. Engl. J. Med. 372(4), $320-330$ (2015).

24. Weber JS, D'Angelo SP, Minor D et al. Nivolumab versus chemotherapy in patients with advanced melanoma who progressed after anti-CTLA-4 treatment (CheckMate 037): a randomised, controlled, open-label, Phase 3 trial. Lancet Oncol. 16(4), 375-384 (2015).

25. Postow MA, Chesney J, Pavlick AC et al. Nivolumab and ipilimumab versus ipilimumab in untreated melanoma. N. Engl. J. Med. 372(21), 2006-2017 (2015).

26. Topalian SL, Sznol M, McDermott DF et al. Survival, durable tumor remission, and long-term safety in patients with advanced melanoma receiving nivolumab. J. Clin. Oncol. 32(10), 1020-1030 (2014).

27. Lian B, Si L, Cui C et al. Phase II randomized trial comparing high-dose IFN- $\alpha 2 b$ with temozolomide plus cisplatin as systemic adjuvant therapy for resected mucosal melanoma. Clin. Cancer Res. 19(16), 4488-4498 (2013).

28. Balch CM, Gershenwald JE, Soong SJ et al. Final version of 2009 AJCC melanoma staging and classification. J. Clin. Oncol. 27(36), 6199-6206 (2009).

29. Wellbrock C, Hurlstone A. BRAF as therapeutic target in melanoma. Biochem. Pharmacol. 80(5), 561-567 (2010).

30. Long GV, Menzies AM, Nagrial AM et al. Prognostic and clinicopathologic associations of oncogenic BRAF in metastatic melanoma. J. Clin. Oncol. 29(10), 1239-1246 (2011).

31. Hodi FS, Corless CL, Giobbie-Hurder A et al. Imatinib for melanomas harboring mutationally activated or amplified KIT arising on mucosal, acral, and chronically sun-damaged skin. J. Clin. Oncol. 31(26), 3182-3190 (2013).

•• Demonstrates that mucosal melanomas with KIT mutations, but not amplifications, may benefit from imatinib.

32. Carvajal RD, Lawrence DP, Weber JS et al. Phase II study of nilotinib in melanoma harboring KIT alterations following progression to prior KIT inhibition. Clin. Cancer Res. 21(10), 2289-2296 (2015).

33. Hayward NK, Wilmott JS, Waddell N et al. Whole-genome landscapes of major melanoma subtypes. Nature 545(7653), 175-180 (2017).

34. Nilsson PJ, Ragnarsson-Olding BK. Importance of clear resection margins in anorectal malignant melanoma. Br. J. Surg. 97(1), 98-103 (2010).

35. Buchanan DJ, Schlaerth J, Kurosaki T. Primary vaginal melanoma: thirteen-year disease-free survival after wide local excision and review of recent literature. Am. J. Obstet. Gynecol. 178(6), 1177-1184 (1998).

36. Iddings DM, Fleisig AJ, Chen SL, Faries MB, Morton DL. Practice patterns and outcomes for anorectal melanoma in the USA, reviewing three decades of treatment: is more extensive surgical resection beneficial in all patients? Ann. Surg. Oncol. 17(1), 40-44 (2010).

37. DeMatos P, Tyler D, Seigler HF. Mucosal melanoma of the female genitalia: a clinicopathologic study of forty-three cases at Duke University Medical Center. Surgery 124(1), 38-48 (1998).

38. Ragnarsson-Olding BK, Nilsson BR, Kanter-Lewensohn LR, Lagerlöf B, Ringborg UK. Malignant melanoma of the vulva in a nationwide, 25-year study of 219 Swedish females: predictors of survival. Cancer 86(7), 1285-1293 (1999).

39. Lee SP, Shimizu KT, Tran LM, Juillard G, Calcaterra TC. Mucosal melanoma of the head and neck: the impact of local control on survival. Laryngoscope 104(2), 121-126 (1994).

40. Manolidis S, Donald PJ. Malignant mucosal melanoma of the head and neck: review of the literature and report of 14 patients. Cancer 80(8), 1373-1386 (1997).

41. Benlyazid A, Thariat J, Temam S et al. Postoperative radiotherapy in head and neck mucosal melanoma: a GETTEC study. Arch. Otolaryngol. Head Neck Surg. 136(12), 1219-1225 (2010).

42. Kelly P, Zagars GK, Cormier JN, Ross MI, Guadagnolo BA. Sphincter-sparing local excision and hypofractionated radiation therapy for anorectal melanoma: a 20-year experience. Cancer 117(20), 4747-4755 (2011).

43. Tchelebi L, Guirguis A, Ashamalla H. Rectal melanoma: epidemiology, prognosis, and role of adjuvant radiation therapy. J. Cancer Res. Clin. Oncol. 142(12), 2569-2575 (2016). 
44. Krengli M, Masini L, Kaanders JH et al. Radiotherapy in the treatment of mucosal melanoma of the upper aerodigestive tract: analysis of 74 cases. A rare cancer network study. Int. J. Radiat. Oncol. Biol. Phys. 65(3), 751-759 (2006).

45. O'reilly A, Larkin J. Checkpoint inhibitors in advanced melanoma: effect on the field of immunotherapy. Expert Rev. Anticancer Ther. 17(7), 647-655 (2017).

46. Achkar T, Tarhini AA. The use of immunotherapy in the treatment of melanoma. J. Hematol. Oncol. 10(1), 88 (2017).

47. Aarsanz H, Laclle A, Lecumberri MJ et al. Immunotherapy in malignant melanoma: recent approaches and new perspectives. Melanoma Manage. 4(1), 39-48 (2017).

48. Schadendorf D, Hodi FS, Robert C et al. Pooled analysis of long-term survival data from Phase II and Phase III trials of ipilimumab in unresectable or metastatic melanoma. J. Clin. Oncol. 33(17), 1889-1894 (2015).

49. Hamid O, Puzanov I, Dummer R et al. Final analysis of a randomised trial comparing pembrolizumab versus investigator-choice chemotherapy for ipilimumab-refractory advanced melanoma. Eur. J. Cancer 86, 37-45 (2017).

50. Ribas A, Hamid O, Daud A et al. Association of pembrolizumab with tumor response and survival among patients with advanced melanoma. JAMA 315(15), 1600-1609 (2016).

51. Larkin J, Chiarion-Sileni V, Gonzalez R et al. Combined nivolumab and ipilimumab or monotherapy in untreated melanoma. N. Engl. J. Med. 373(1), 23-34 (2015).

52. Wolchok JD, Chiarion-Sileni V, Gonzalez R et al. Overall survival with combined nivolumab and ipilimumab in advanced melanoma. N. Engl. J. Med. 377(14), 1345-1356 (2017).

53. Shoushtari AN, Munhoz RR, Kuk D et al. The efficacy of anti-PD-1 agents in acral and mucosal melanoma. Cancer 122(21), 3354-3362 (2016).

54. Tumeh PC, Harview CL, Yearley JH et al. PD-1 blockade induces responses by inhibiting adaptive immune resistance. Nature 515(7528), 568-571 (2014).

55. Song H, Wu Y, Ren G, Guo W, Wang L. Prognostic factors of oral mucosal melanoma: histopathological analysis in a retrospective cohort of 82 cases. Histopathology 67(4), 548-556 (2015).

56. Robert C, Karaszewska B, Schachter J et al. Improved overall survival in melanoma with combined dabrafenib and trametinib. N. Engl. J. Med. 372(1), 30-39 (2015).

57. Carvajal RD, Antonescu CR, Wolchok JD et al. KIT as a therapeutic target in metastatic melanoma. JAMA 305(22), 2327-2334 (2011).

58. Guo J, Si L, Kong Y et al. Phase II, open-label, single-arm trial of imatinib mesylate in patients with metastatic melanoma harboring c-Kit mutation or amplification. J. Clin. Oncol. 29(21), 2904-2909 (2011).

59. Kalinsky K, Lee S, Rubin KM et al. A Phase II trial of dasatinib in patients with locally advanced or stage IV mucosal, acral, or vulvovaginal melanoma: a trial of the ECOG-ACRIN Cancer Research Group (E2607). Cancer 123(14), 2688-2697 (2017).

60. Johnson DB, Puzanov I. Treatment of NRAS-mutant melanoma. Curr. Treat. Options Oncol. 16(4), 15 (2015).

61. Ascierto PA, Schadendorf D, Berking C et al. MEK162 for patients with advanced melanoma harbouring NRAS or Val600 BRAF mutations: a non-randomised, open-label Phase II study. Lancet Oncol. 14(3), 249-256 (2013).

62. Chapman PB, Einhorn LH, Meyers ML et al. Phase III multicenter randomized trial of the Dartmouth regimen versus dacarbazine in patients with metastatic melanoma. J. Clin. Oncol. 17(9), 2745-2751 (1999).

63. Apetoh L, Ladoire S, Coukos G, Ghiringhelli F. Combining immunotherapy and anticancer agents: the right path to achieve cancer cure? Ann. Oncol. 26(9), 1813-1823 (2015).

64. Robert C, Thomas L, Bondarenko I et al. Ipilimumab plus dacarbazine for previously untreated metastatic melanoma. N. Engl. J. Med. 364(26), 2517-2526 (2011).

65. Sherrill B, Wang J, Kotapati S, Chin K. Q-TWiST analysis comparing ipilimumab/dacarbazine vs placebo/dacarbazine for patients with stage III/IV melanoma. Br. J. Cancer 109(1), 8-13 (2013).

66. Eggermont AM, Chiarion-Sileni V, Grob JJ et al. Adjuvant ipilimumab versus placebo after complete resection of high-risk stage III melanoma (EORTC 18071): a randomised, double-blind, Phase III trial. Lancet Oncol. 16(5), 522-530 (2015).

67. Weber J, Mandala M, Del Vecchio M et al. Adjuvant nivolumab versus ipilimumab in resected stage III or IV melanoma. N. Engl. J. Med. 377(19), 1824-1835 (2017).

68. Joensuu H, Vehtari A, Riihimäki J et al. Risk of recurrence of gastrointestinal stromal tumour after surgery: an analysis of pooled population-based cohorts. Lancet Oncol. 13(3), 265-274 (2012).

69. Long GV, Hauschild A, Santinami M et al. Adjuvant dabrafenib plus trametinib in stage III BRAF-mutated melanoma. N. Engl. J. Med. 377(19), 1813-1823 (2017). 
